\title{
Meloidogyne Virulence Locus Molecular Marker for Characterization of Selected Mi-Virulent Populations of Meloidogyne spp. Is Correlated with Several Genera of Betaproteobacteria
}

\author{
Laura Cortada, Hiromichi Sakai, Soledad Verdejo-Lucas, and Takayuki Mizukubo
}

First and third authors: IRTA, Patología Vegetal, Crta. de Cabrils Km 2, 08348 Cabrils, Barcelona, Spain; and second and fourth authors:

National Agricultural Research Center, Kannondai 3-1-1, Tsukuba, Ibaraki, 305-8666, Japan.

Accepted for publication 8 October 2010.

\begin{abstract}
Cortada, L., Sakai, H., Verdejo-Lucas, S., and Mizukubo, T. 2011. Meloidogyne virulence locus molecular marker for characterization of selected Mi-virulent populations of Meloidogyne spp. is correlated with several genera of betaproteobacteria. Phytopathology 101:410-415.

Resistance to root-knot nematodes in tomato is conferred by the $M i$ resistance gene to the three most important species of Meloidogyne: $M$. arenaria, $M$. incognita, and $M$. javanica. Nevertheless, the $M i$ gene is unable to inhibit the reproduction of selected and naturally $M i$-virulent populations of root-knot nematodes. As pathogenicity assays are time consuming, molecular markers were developed for the easy identification of Mi-virulent populations of Meloidogyne. The sequence characterized

amplified region-Meloidogyne virulence locus (MVC) molecular marker is reported to differentiate $M i$-avirulent and naturally $M i$-virulent from selected $M i$-virulent populations. This marker was used to compare acquired virulence in populations of $M$. javanica from Spain. The original populations used to develop the MVC marker were included as control for reference. Results showed that this marker did not amplify genomic DNA extracted from single juveniles or females of any of the populations tested either from Spain or Japan. In silico analyses performed with the recently published complete genome of $M$. incognita, indicated that the MVC marker is not correlated to a MVC or to any eukaryotic organism but to several betaproteobacteria genus from the family Comamonadaceae.
\end{abstract}

Resistance to Meloidogyne arenaria, $M$. incognita, and $M$. javanica in tomato is conferred by a single dominant gene named $M i$ (30). In nematology, virulence is defined as the ability of a nematode to infect and reproduce on a resistant plant, evolving into a fertile female (22). Populations able to overcome the resistance provided by the $M i$ gene are classified as naturally or selected $M i$-virulent. The later appear after repeated exposure to the $M i$ gene $(5,9,14,28,31)$, whereas naturally $M i$-virulent populations reproduce significantly on resistant tomato cultivars despite never having been exposed to the $M i$ gene $(15,19,20,33)$. Independent of their origin, $M i$-virulent populations negate the use of resistance as a management option for root-knot nematodes.

The gene-for-gene model postulates that for every avirulence gene $(A v r)$ present in the pathogen, there is a resistant gene $(R)$ in the host that prevents infection. So far, Avr genes from nematodes have not been cloned (32) although there is genetic evidence that avirulence in certain species of plant-parasitic nematodes is transmitted to the progeny as a single dominant trait (13). This Avr-R gene model has been proved for Globodera rostochiensis and the $R$ gene $H 1$ (2), for $M$. hapla and the bean cultivar NemaSnap (8), and more recently for the $G p-R B P-1$ protein from $G$. pallida and the Gpa2 gene (23). Evidence to confirm this model in the rootknot nematode-resistant plant pathosystem are scarce (4), because the strictly parthenogenetic mode of reproduction of $M$. arenaria, $M$. incognita, and $M$. javanica does not permit the studying of the segregation of the $M i$-virulent alleles ( $a v r$ ) in the progeny of these species. In 2001, Xu et al. (33) developed the

Corresponding author: L. Cortada; E-mail address: lauracortada78@gmail.com

* The $\boldsymbol{e}$-Xtra logo stands for "electronic extra" and indicates that the online version contains a supplemental figure.

doi:10.1094/PHYTO-04-10-0123

(c) 2011 The American Phytopathological Society sequence characterized amplified region-polymerase chain reaction (SCAR-PCR) Meloidogyne virulence correlated (MVC) molecular marker to differentiate selected $M i$-virulent populations of Meloidogyne from naturally $\mathrm{Mi}$-virulent and $\mathrm{Mi}$-avirulent populations; and it was suggested that selected $M i$-virulent nematode isolates originated by similar mutational events and that they diverged genetically from those naturally $M i$-virulent which have never been exposed to the selection pressure of the $M i$ gene.

The recent publication of the complete genome of $M$. incognita and M. hapla (4) offered the opportunity to correlate the sequences of the MVC locus with the genome of these species. In silico analyses performed with the original MVC-allelic sequences (GenBank accession nos. AB047761.1 to AB047767.1) did not reveal a correlation between these sequences and the genome of any nematode species including that of Caenorhabditis elegans or any eukaryotic organism. However, a close homology $(E$ values $=0$; maximum identities $=71$ to $88 \%)$ was found with the genome of seven genera of betaproteobacteria belonging to the family Comamonadaceae: Acidovorax spp. (accession nos. CP001392.1 and CP000512.1), Delftia spp. (accession no. CP000884.1), Polaromonas spp. (accession no. CP000316.1), Rhodopherax spp. (accession no. CP000267.1), Variovorax spp. (accession no. CP001635.1), and Verminephrobacter spp. (accession no. CP000542.1). The objective of the present study was to demonstrate that the MVC molecular marker is correlated to the genome of a prokaryotic organism and not to a root-knot nematode virulence locus.

\section{MATERIALS AND METHODS}

Molecular analyses were performed with seven populations of the genus Meloidogyne from different geographic origins: (i) two Japanese laboratory-selected $M i$-virulent populations, $M$. javanica (MJON-VI) and M. incognita (MIYN-VI) (33); and (ii) five 
populations from north-eastern Spain that differed in their ability to reproduce on $M i$-resistant tomatoes: three $M$. javanica fieldselected $M i$-virulent populations (PG-76-P3, Brigeor-P3, and Monika-P3), one Mi-avirulent (Durinta-P3) (28), and one naturally $M i$-virulent population (MJ-27) (19). Characteristics of the populations are described in Table 1. All the $M i$-virulent populations from Japan have been maintained on resistant tomato plants (cv. Momotaro) since they were first identified as $\mathrm{Mi}$ virulent (T. Narabu, personal communication). In the case of the Spanish populations, they have been maintained on resistant tomato cv. Monika and bioassays indicated that the ability of these populations to reproduce on $M i$-resistant plants has remained stable along this period (29).
Water samples. Water samples were obtained from a potted resistant tomato plant (cv. Momotaro) infected by the MIYN-VI population. The infected plant was watered with distilled sterilized water and the draining water (150 $\mathrm{ml}$ approximately) was collected on a beaker and stored at $4{ }^{\circ} \mathrm{C}$, to restrict the growth of microorganisms. Three types of water treatments were investigated (Fig. 1A): (i) the drained water directly collected from the pot, which was called "nonfiltered water" (NF); (ii) the drained water filtered through a $5 \mu \mathrm{m}$ pore-sieve of nylon to retain nematode second-stage juveniles (J2) and eggs (from now on referred as $5 \mathrm{~F}$ sample), and (iii) an aliquot of the $5 \mathrm{~F}$ sample filtered again through a $0.2 \mu \mathrm{m}$ cellulose acetate filter (AstroPore Disk Capsule CALC. 20, 2,5 CMD; Fuji Film, Kanagawa, Japan)

TABLE 1. Characteristics of the populations of Meloidogyne spp. used for the molecular analyses

\begin{tabular}{|c|c|c|c|c|c|}
\hline Species & Population & Origin & $M i$-virulence ${ }^{\mathrm{a}}$ & Host & Reference \\
\hline \multirow[t]{6}{*}{ M. javanica } & MJ-27 & Barcelona, Spain & VI-natural & $M i$-tomato cultivar & (19) \\
\hline & PG-76-P3 & Barcelona, Spain & VI-selected* & $M i$-tomato rootstock & (28) \\
\hline & Brigeor-P3 & Barcelona, Spain & VI-selected $*$ & $M i$-tomato rootstock & (28) \\
\hline & Monika-P3 & Barcelona, Spain & VI-selected $*$ & $M i$-tomato cultivar & (28) \\
\hline & MJON-VI & Okinawa, Japan & VI-selected $* *$ & $M i$-tomato cultivar & (33) \\
\hline & Durinta-P3 & Barcelona, Spain & AV & $M i$-tomato cultivar & (28) \\
\hline M. incognita & MIYN-VI & Yamanashi, Japan & VI-selected* & $M i$-tomato cultivar & (33) \\
\hline
\end{tabular}

a VI (Mi-virulent) and AV (Mi-avirulent); * indicates $M i$-virulent field selected populations and ** indicates $M i$-virulent laboratory selected population.

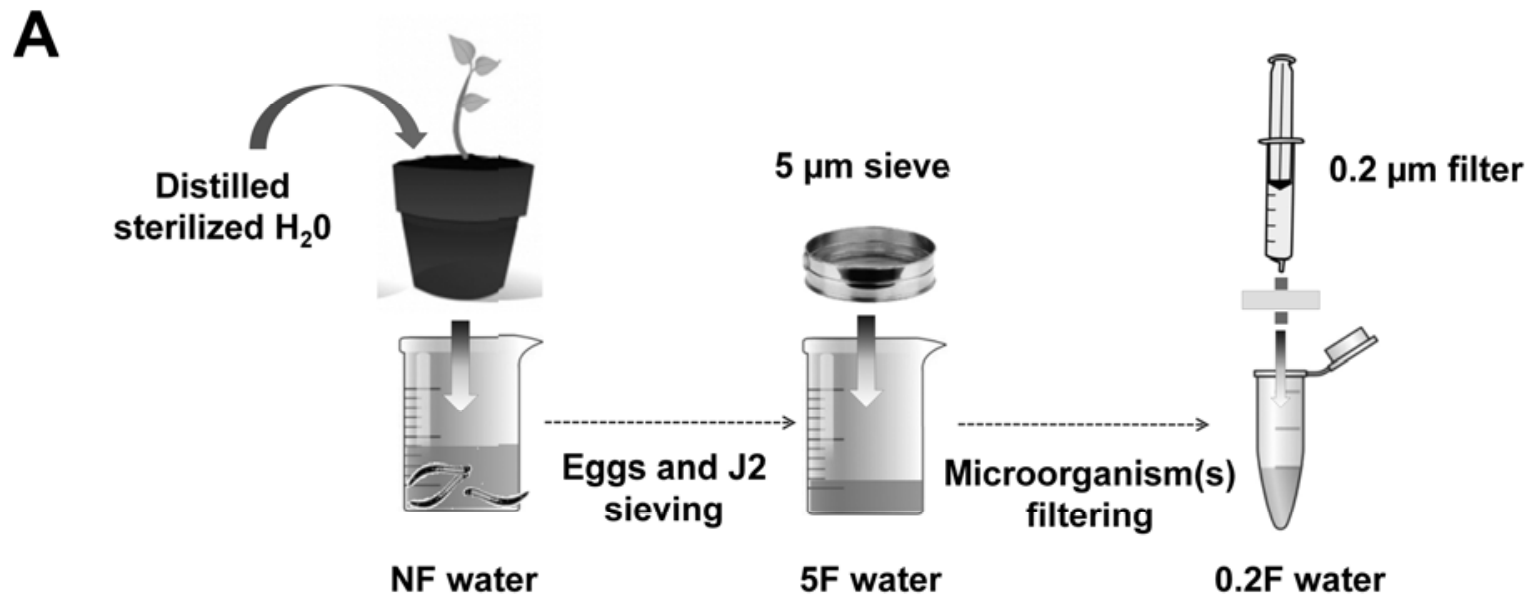

B

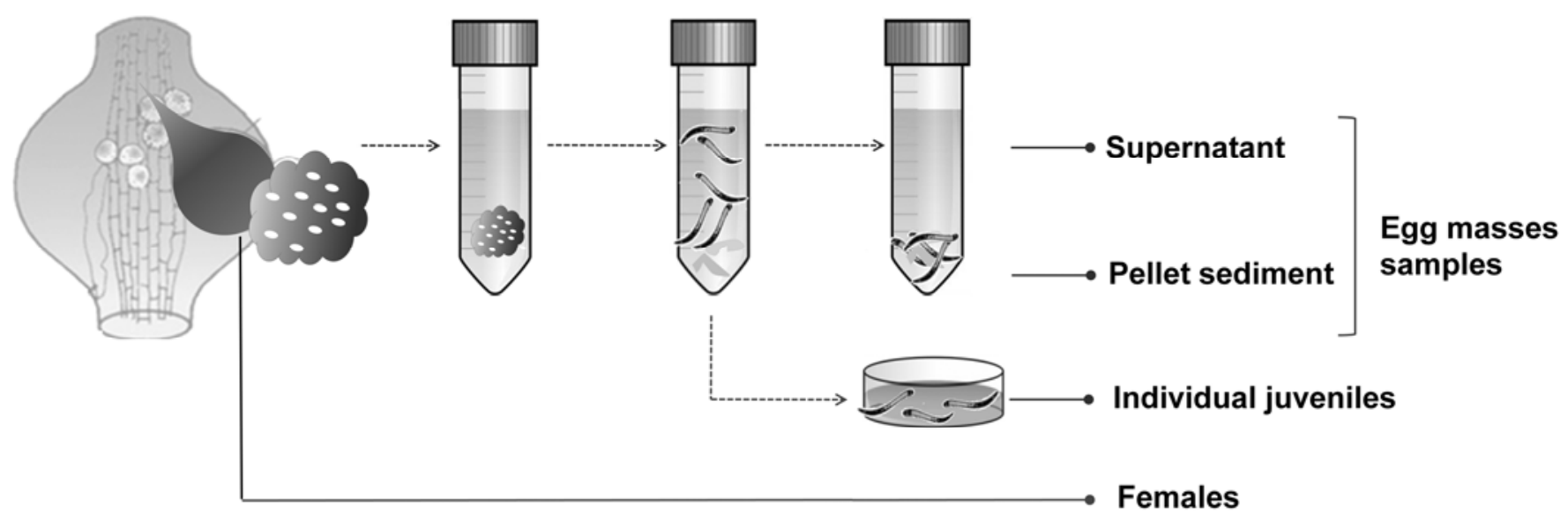

Fig. 1. Types of samples used for DNA extraction. A, Water drained directly from a potted resistant tomato plant infected by the selected $M i$-virulent Meloidogyne incognita population (MIYN-VI). Water treatments: NF, draining water directly collected from the pot; $5 \mathrm{~F}$, originated from the NF water sample filtered through a $5 \mu \mathrm{m}$ pore-sieve of nylon to retain second-stage juveniles (J2) and eggs; $0.2 \mathrm{~F}$, obtained by the filtration of an aliquot of the $5 \mathrm{~F}$ water through a $0.2 \mu \mathrm{m}$ cellulose acetate filter to retain microorganism(s). B, Egg masses, females, and J2 samples from the M. javanica (MJON-VI) and the MIYN-VI selected Mi-virulent populations. 
that was identified as $0.2 \mathrm{~F}$ sample; the last filtration step was performed in order to retain any possible microorganism(s) present in the sample (i.e., bacteria or fungal spores). For DNA extraction, one milliliter of the NF, $5 \mathrm{~F}$, and $0.2 \mathrm{~F}$ water samples were pipetted separately into 1.5-ml Eppendorf tubes and spun at 13,000 rpm for $20 \mathrm{~min}$ at $4^{\circ} \mathrm{C}$. Three samples of each were analyzed. Supernatant was removed and $4 \mu \mathrm{l}$ of $0.1 \%$ SDS lysis buffer (24) was added to the tubes; samples were incubated at $50^{\circ} \mathrm{C}$ in a thermal cycler for $2 \mathrm{~h}$ followed by one cycle at $95^{\circ} \mathrm{C}$ for $10 \mathrm{~min}$ and $196 \mu \mathrm{l}$ of sterilized water was added and vortexed (24).

Egg mass samples. Eight egg masses (EMs) of the MJON-VI population were used for molecular analyses. All the EMs were placed individually into screw-cap vials filled with $2 \mathrm{ml}$ of sterile distilled water, and were left at room temperature to allow hatching of the $\mathrm{J} 2$ from the eggs (Fig. 1B). Vials were then vigorously shaken and $\mathrm{J} 2 \mathrm{~s}$ were left to settle at the bottom of the vials for $3 \mathrm{~h}$. For every sample, $1 \mathrm{ml}$ from each supernatant and pelleted sediment phase (Fig. 1B) was pipetted separately into $1.5-\mathrm{ml}$ Eppendorf tubes, spun, and processed for DNA extraction as described for water samples.

Juvenile and female samples. DNA extraction was performed for 20 single $\mathrm{J} 2$ of the MJON-VI population collected from vials containing a single EM each (Fig. 1B). Juveniles were individually hand-picked with the aid of a sterilized needle and placed on a 5- $\mu$ l drop of sterilized Milli-Q water on a glass slide where $\mathrm{J} 2$ were crushed with a sterilized filter paper chip ( $1 \mathrm{~mm} \times$ $1 \mathrm{~mm})$. Molecular analyses were additionally performed over 10 females of the MJON-VI population, three females of the MIYNVI population, and 40 from each of the five nematode populations from Spain. For this purpose, females were dissected from infected roots of resistant tomato plants (cv. Momotaro and cv. Monika for Japanese and Spanish populations, respectively) with forceps under a stereo microscope. All females were stored at $-80^{\circ} \mathrm{C}$ until DNA was extracted; as for $\mathrm{J} 2$, females were also squashed prior to DNA extraction. Paper chips with either the crushed $\mathrm{J} 2$ or the females were introduced individually into a 0.5-ml Eppendorf tube; DNA extraction and incubation were as indicated previously. DNA extraction from the females from the Spanish populations was performed according to Cenis (7).

PCR analyses. PCR conditions were those described by Xu et al. (33) at a final volume of $40 \mu \mathrm{l}$, including $20 \mu \mathrm{l}$ of DNA template (0.001\% SDS final concentration), 10× PCR buffer, and 0.5 units of Ex Taq DNA polymerase (Takara Biomedicals, Otsu, Japan); amplification was held for 40 cycles. Molecular analyses included the co-dominant marker MVC-F3/R2 that amplifies a fragment of $\approx 873 \mathrm{bp}$ in all types of populations ( $M i$-avirulent, selected $M i$-virulent and naturally $M i$-virulent) and the dominant marker MVC-VF/R1 that exclusively amplifies a $\approx 1,032$-bp band in the genome of the selected $M i$-virulent populations (33). The

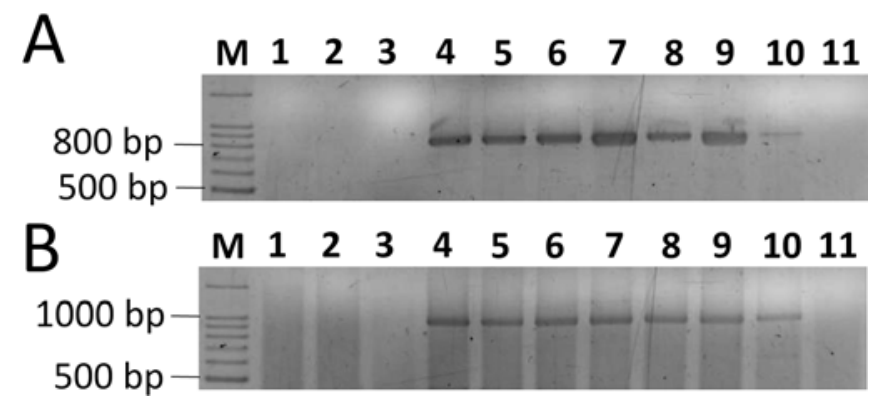

Fig. 2. Meloidogyne virulence locus (MVC) marker profile of draining-water samples collected from a potted plant infected by a laboratory selected $M i$ virulent population of Meloidogyne incognita (MIYN-VI). A, Co-dominant molecular marker MVC-F3/R2. B, Dominant molecular marker MVC-VF/R1. Lanes: (1 to 3) 0.2F water, (4 to 6) 5F water, (7 to 9) NF water, (10) Supernatant of egg mass no. $3\left(\mathrm{EM}_{3}\right)$, and (11) Mili-Q sterilized water. Lane $\mathrm{M}$ indicates $100-\mathrm{bp}$ DNA ladder.
MVC-F3/R2 amplified products were digested overnight with the NdeI restriction enzyme (Takara Biomedicals) following the manufacturer's protocol. Nematode DNA extraction was tested by PCR amplification of the ITS region (10). For all reactions, $5 \mu \mathrm{l}$ of the PCR products was resolved on $1.5 \%$ agarose gels in $0.5 \times$ TBE buffer. Electrophoresis was at $90 \mathrm{~V}$ for $60 \mathrm{~min}$. All PCR reactions were repeated twice.

DNA sequencing. PCR products obtained with the MVCVF/R1 marker were cut from the gel and purified with a MinElute Gel Extraction Kit (Qiagen). DNA labeling was performed using BigDye Terminator version 1.1 (Applied Biosystems) followed by purification with Dye Ex 2.0 Spin Kit (Qiagen). Sequencing was performed in an ABI PRISM 3100 Genetic Analyzer (Applied Biosystems). DNA sequences were manually corrected using Bioedit version 7.0.9 program. The resulting sequences were then compared with the sequences available from public databases through a nucleotide BLAST program (http://blast.ncbi.nlm.nih.gov/Blast.cgi).

\section{RESULTS}

Amplification with the MVC molecular markers in the absence of nematodes. The expected DNA fragments were consistently amplified with the co-dominant MVC-F3/R2 and the dominant MVC-VF/R1 markers in all the $\mathrm{NF}$ and $5 \mathrm{~F}$ water samples analyzed but not in the $0.2 \mathrm{~F}$ samples: that is, in samples without nematodes (Fig. 2A and B) but presumably containing microorganisms that were retained on the $0.2 \mu \mathrm{m}$ cellulose filter. DNA amplification for both markers also occurred in the supernatant phase obtained from the aqueous suspensions of individual EMs (Fig. 3A, lanes 1 to 8; Fig. 3B, lanes 1, 2, 4, and 5). Samples from the sedimented pellet phase that contained J2 and the remains of the gelatinous matrix (GM) from the EMs (Fig. 3) showed similar results.

The MVC-VF/R1 PCR-products obtained from the supernatant phase of the $\mathrm{EM}_{4}$-water suspension $\left(\mathrm{MVC} / \mathrm{S}-\mathrm{EM}_{4}\right)$ (Fig. 3B, lane 2) were sequenced. A BLAST search on the public databases revealed that the 1,032-bp amplified fragment presented a high homology to the seven original MVC-allelic sequences (GenBank accession no. $\mathrm{AB} 047761$ to $\mathrm{AB} 047767)$, but also to a ribosome small subunit-dependent GTPase A (RsgA) and a pterin-4-alpha carbinolamine dehydratase (PCD) from the betaproteobacteria species Verminephrobacter eiseniae EF01-2 (score $=951, E$ value $=0$ ), Acidovorax avenae subsp. citrulli AAC00-1 (score = $771, E$ value $=0)$ and Variovorax paradoxus $\mathrm{S} 110$ (score $=574, E$ value $=3 e-160$ ); to a Ste24 endopeptidase (Ste24) and a transcriptional coactivator/pterin dehydratase from the species Acidovorax ebreus TPSY (score $=767, E$ value $=0$ ) and Delftia acidovorans SPH-1 (score $=618, E$ value $=3 e-173$ ); and to a ribosome associated GTPase EngC and a PCD from the species Rhodoferax ferrireducens T118 (score $=479, E$ value $=2 e-131)$ and the genus Polaromonas sp. JS666 (score $=446 ; E$ value $=$ $1 e-121)$. In addition, the $\mathrm{MVC}_{\mathrm{S}-\mathrm{EM}_{4}}$ sequence revealed a mutation in the target site of the restriction enzyme NdeI which could explain why no restriction fragments were obtained after digestion of the co-dominant PCR products from single $\mathrm{J} 2$ of the selected $M i$-virulent population MJON-VI.

Absence of amplification of the MVC molecular markers in DNA from $\mathbf{J} 2$ and females. The co-dominant MVC-F3/R2 marker did not amplify any DNA fragment in any of the J2 individually analyzed from the MJON-VI population, or in a pool of DNA composed of DNA extractions from 10 single $\mathrm{J} 2$ from two independent EMs (10 J2/EM) of the same population, or in the two adult fertile females of the MIYN-VI population (Fig. 4). Similarly, the co-dominant MVC-F3/R2 marker did not amplify any DNA fragment when using the three field-selected $\mathrm{Mi}$ virulent populations PG-76-P3, Brigeor-P3, and Monika-P3, the naturally $M i$-virulent population (MJ-27), and the $M i$-avirulent population Durinta-P3 as DNA template (Fig. 5A and B). For all 
nematode samples, amplification of the ITS region was successfully performed in order to check DNA quality (data not shown).

\section{DISCUSSION}

Recent advances in the sequencing of the complete genome of $M$. incognita and $M$. hapla and of several betaproteobacteria species from the family Comamonaceae, together with the experimental evidence presented in this paper indicates that the MVC locus is not related to a root-knot nematode Avr gene. The SCARMVC molecular marker repeatedly failed to amplify DNA from $\mathrm{J} 2$ and females from $M i$-avirulent, laboratory and field selected $M i$-virulent and naturally $M i$-virulent populations of $M$. incognita and $M$. javanica, including two of the Japanese populations originally used to develop this marker (33). Conversely, the genomic DNA amplified by the primer pair MVC-VF/R1 was closer to a prokaryotic than to a eukaryotic genome. Amplification of DNA from a microorganism(s) and not from the nematode itself, would explain why the results reported by $\mathrm{Xu}$ et al. (33) have been considered as nonreproducible for non-Asiatic isolates of Meloidogyne spp. (3). In agreement with this hypothesis, the results presented in this paper could also explain why the MVC molecular markers only amplified DNA from minipreps of EMs, but not from single $\mathrm{J} 2$ in the original paper (33). Possibly, no amplification occurred in $\mathrm{J} 2$ as microorganism(s) adhered to the cuticle surface would have been washed away while hand picking them from the sterile water suspension; in a similar way, adult females might have been protected inside the root tissue from attachment of the microorganism(s). As Xu et al. (33) did not find any significant correlations at the nucleic acid level for the MVC-allelic sequences in the public databases available in 2001, they concluded that the MVC locus was intimately related to the acquired $\mathrm{Mi}$-virulence in Meloidogyne spp. The recent sequencing of the genomes of Acidovorax avenae subsp. citrulli AAC00-1, A. ebreus TPSY, Delftia acidovorans SPH-1, Polaromonas sp. JS666, Rhodoferax ferrireducens T118, Variovorax paradoxus S110, and Verminephrobacter eiseniae EF01-2 (DOE Joint Genome Institute at http://www.jgi.doe.gov/ genome-projects/pages/projects.jsf; 6,25) allowed us to detect the homology between the MVC-amplified sequences and those from diverse proteobacterial enzymes.

The amplification of several genomic bands with the codominant molecular marker in the aqueous suspensions of the
EMs suggests that the microorganism(s) were highly polymorphic or that different species of microorganisms were amplified. Further studies will be necessary to determine the microbiologic origin of the genomic DNA amplified by the MVC molecular marker in the selected $M i$-virulent populations from Japan. Regardless of the origin or the identity of the microorganism(s) detected, they must have been very abundant as DNA amplification occurred readily in all the aqueous suspensions samples from the EMs and the drained-water samples from the potted plant.

It is well known that bacteria play an important role in the protection of nematode eggs against microorganisms (18). Up to 70 bacterial isolates have been reported in association with the GM of the EMs of M. hapla from infected tomato roots, with Acidovorax being the second most abundant genus (17). Although in silico analyses established the highest similarity with $V$. eiseniae EFO1-2, an endosymbiont of the earthworm Eisenia foetida (21), it is unlikely that the MVC-amplified genomic DNA corresponded to an endosymbiont bacterium as no amplification occurred in individual $\mathbf{J} 2$. Homology was also found to the strains A. avenae subsp. citrulli AAC00-1, A. ebreus TYPS, and D. acidovorans $\mathrm{SPH}-1$. These bacterial genera are frequently present in waste water treatment plants, aquatic environments, soil, sludge, or siderurgic-polluted environments (11), and some species can be found as a group of plant pathogens $(16,21)$. The enzymes showing the highest homology with the sequence amplified from the aqueous suspension of an EM present a zinc-

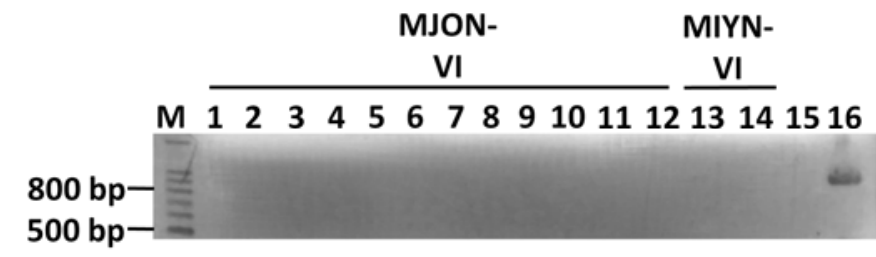

Fig. 4. Meloidogyne virulence locus (MVC)-F3/R2 marker profile of the $M i$ virulent laboratory selected population of Meloidogyne javanica (MJON-VI) and field selected population M. incognita (MIYN-VI) from Japan. Lanes: (1 to 10$)$ individual juveniles (J2) from one egg mass $\left(\mathrm{EM}_{1}\right)$ of the MJON-VI population, (11) pool of DNA from $10 \mathrm{~J} 2$ of $\mathrm{EM}_{1}$, (12) pool of DNA from 10 $\mathrm{J} 2$ of $\mathrm{EM}_{2}$, (13 to 14) individual females of the MIYN-VI population, (15) Mili-Q sterilized water, and (16) supernatant of $\mathrm{EM}_{3}$. Lane $\mathrm{M}$ indicates 100-bp DNA ladder.

(S)

(P)
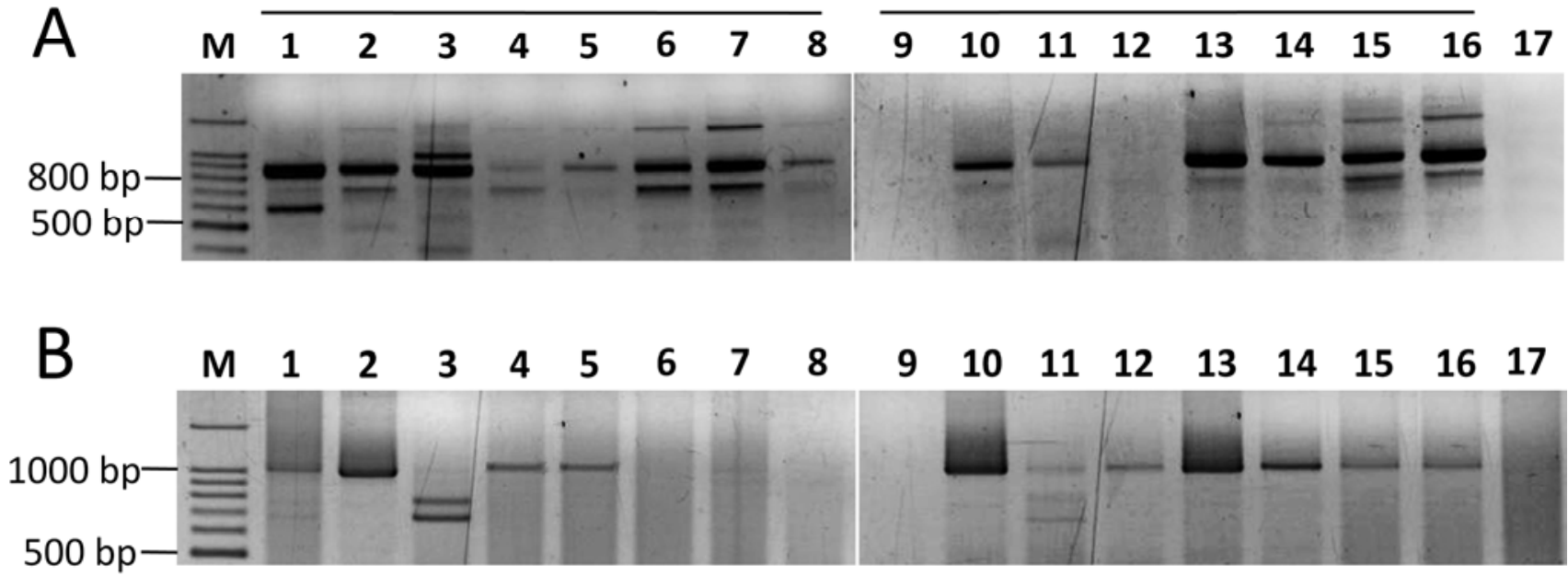

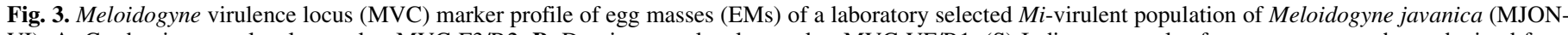

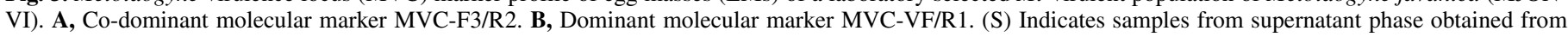

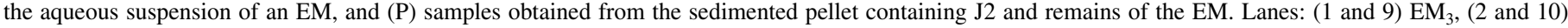

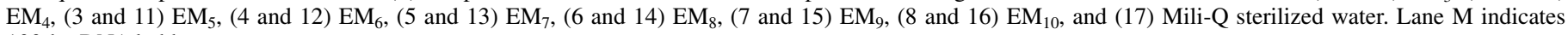
100-bp DNA ladder. 
binding motif and have been described in several bacteria and plants.

Genetic differences were found between the MVC/S-EM4 and the MJON-VI sequences. Despite the MVC/S-EM4 sequence having been obtained from a MJON-VI infected plant, this sequence was genetically closer to betaproteobacteria proteins than to the original MJON-VI sequence (accession no. AB047762). The fact that NdeI was able to digest the genomic sequences of the MJON-VI populations in $\mathrm{Xu}$ et al. (33) experiments but not in the ones described here, can be explained by a mutation in the target sequence of the restriction enzyme. The maintenance and replication of this population originating from a single EM for the last 10 years may have changed the bacterial community associated to the population MJON-VI or selected a mutated bacterial strain through several generations.

Therefore, the MVC molecular marker could not be used to characterize at the molecular level selected $M i$-virulence in populations of $M$. javanica acquired after repeated exposure to the $M i$ gene in the field (28). Many efforts have been made during the past years to characterize $M i$-virulent populations through molecular approaches $(1,26)$. The protein $M A P-1$, the first candidate coding for a nematode Avr gene (27), was found in $\mathrm{Mi}$ avirulent populations of $M$. arenaria, $M$. incognita, and $M$. javanica subjected to the $M i$-selection pressure although not in $M$. hapla or $M$. fallax. Nevertheless, no functional evidence correlating the $A v r$ candidate protein $M A P-1$ to virulence or avirulence has been established, as this protein did not reveal genetic polymorphisms between two closely related $M i$-virulent and $M i$-avirulent isolates of $M$. javanica (12). Recently, the new candidate $C g-1$ gene has been related to avirulence in $M$. javanica, as $C g-1$ is required in the nematode for the expression of the $\mathrm{Mi}$-mediated resistance response in the plant (13). The $\mathrm{Cg}$ 1 marker has also been detected in the three most important species of Meloidogyne affected by the Mi-mediated resistance, suggesting that this gene was probably present in a common nematode ancestor $(12,13)$. However, the $C g-1$ has been amplified from some $M i$-virulent isolates of $M$. javanica and the copies of $C g-1$ in $M$. incognita are different in sequence from those in $M$. javanica (V. M. Williamson, personal communication), suggesting that $C g-1$ does not explain all virulence to $M i$. Hence a universal molecular marker that can distinguish root-knot nematode strains that are $\mathrm{Mi}$-virulent from those that are $\mathrm{Mi}$ -
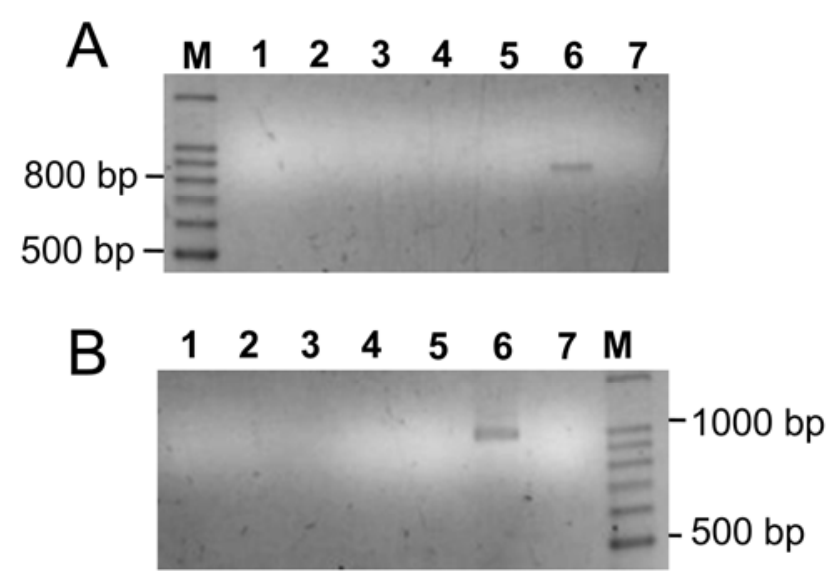

Fig. 5. Meloidogyne virulence locus (MVC) marker profile of the field selected $M i$-virulent populations of $M$. javanica (PG-76-R3, Brigeor-P3, and Monika-P3), the naturally $M i$-virulent $M$. javanica population (MJ-27), and the $M i$-avirulent $M$. javanica (Durinta-P3), all from north-eastern Spain; and the laboratory selected $M i$-virulent $M$. javanica population (MJON-VI) from Japan. A, Co-dominant molecular marker MVC-F3/R2. B, Dominant molecular marker MVC-VF/R1. Lanes: (1) PG-76-R3, (2) Brigeor-P3, (3) Monika-

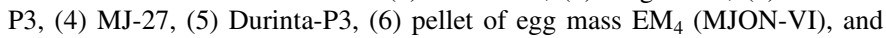
(7) Mili-Q sterilized water. Lane M indicates 100-bp DNA ladder. avirulent has not been obtained yet. Under this perspective, virulence will still need to be determined by pathogenicity assays until new molecular markers are generated.

\section{ACKNOWLEDGMENTS}

L. Cortada acknowledges the Spanish Instituto Nacional de Investigaciones Agrarias (INIA) for support through a pre-doctoral grant. Thanks are given to I. Iwahori and T. Narabu for providing the nematode populations, P. Manzano, F. J. Sorribas, and C. Ornat for critical reading of the manuscript, and C. Lorente for suggestions.

\section{LITERATURE CITED}

1. Abad, P., Favery, B., Rosso, M., and Castagnone-Sereno, P. 2003. Rootknot nematode parasitism and host response: Molecular basis of a sophisticated interaction. Mol. Plant Pathol. 4:217-224.

2. Bakker, J. 2002. Durability of resistance against potato cyst nematodes. Euphytica 124:157-162.

3. Bleve-Zacheo, T., Melillo, M. T., and Castagnone-Sereno, P. 2007. The contribution of biotechnology to root-knot nematode control in tomato plants. Pest Technol. 1:1-16.

4. Bird, D. McK., Williamson, V. M., Abad, P., McCarter, J., Danchin, E. G. J., Castagnone-Sereno, P., and Opperman, C. H. 2009. The genomes of root-knot nematodes. Annu. Rev. Phytopathol. 47:333-351.

5. Bost, S. C., and Triantaphhyllou, A. C. 1982. Genetic basis of the epidemiological effects of resistance to Meloidogyne incognita in the tomato cultivars Small Fry. J. Nematol. 14:540-544.

6. Byrne-Bailey, K. G., Weber, K. A., Chair, A. H., Bose, S., Knox, T., Spanbauer, T. L., Chertkov, O., and Coates, J. D. 2010. Completed genome sequence of the anaerobic iron-oxidizing bacterium Acidovorax ebreus strain TPSY. J. Bacteriol. 192:1475-1476.

7. Cenis, J. L. 1993. Identification of four Meloidogyne spp. by random amplified polymorphic DNA (RAPD-PCR). Phytopathology 83:76-78.

8. Chen, P. C., and Roberts, P. A. 2003. Genetic analysis of (a)virulence in Meloidogyne hapla to resistance in bean (Phaseolus vulgaris). Nematology 5:687-697.

9. Eddaoudi, M., Ammati, M., and Rammah, A. 1997. Identification of resistance breaking populations of Meloidogyne on tomatoes in Morocco and their effect on new sources of resistance. Fundam. Appl. Nematol. 20:285-289.

10. Ferris, V. R., Ferris, J. M., and Faghihi, J. 1993. Variation in spacer ribosomal DNA in some cyst-forming species of plant parasitic nematodes. Fundam. Appl. Nematol. 16:177-184.

11. Freitas, D. B., Lima-Bittencourt, C. I., Reis, M. P., Costa, P. S., Assis, P. S., Chartone-Souza, E., and Nascimento, A. M. A. 2008. Molecular characterization of early colonizer bacteria from wastes in a steel plant. Lett. Appl. Microbiol. 47:241-249.

12. Gleason, C. A. 2003. Comparison of two strains of Meloidogyne javanica differing in virulence on tomato with the resistance gene $M i$, and identification of a polymorphism that correlates with avirulence. Ph.D. thesis, University of California, Davis.

13. Gleason, C. A., Liu, Q. L., and Williamson, V. M. 2008. Silencing a candidate nematode effector gene corresponding to the tomato resistance gene $M i-1$ leads to acquisition of virulence. Mol. Plant-Microbe Interact. 21:576-585.

14. Jarquin-Barberena, H., Dalmasso, A., de Guiran, G., and Cardin, M. C. 1991. Acquired virulence in the plant parasitic nematode Meloidogyne incognita. 1 Biological analysis of the phenomenon. Rev. Nematol. $14: 261-275$.

15. Kaloshian, I., Williamson, V. M., Miyao, G., Lawn, D. A., and Westerdahl, B. B. 1996. "Resistance-breaking" nematodes identified in California tomatoes. Calif. Agric. 50:18-19.

16. Kardenavis, A. A., Kapley, A., and Purohit, H. J. 2007. Simultaneous nitrification and denitrification by diverse Diaphorobacter sp. Appl. Microbiol. Biot. 77:403-409.

17. Kok, C. J., Papert, A., and Hok-a-Hin, C. H. 2001. Microflora of Meloidogyne egg masses: Species composition, population density and effect on the biological control agent Verticillium chlamydosporium (Goddard). Nematology 3:729-734.

18. Orion, D., Kritzman, G., Meyer, S. L. F., Erbe, E. F., and Chitwood, D. J. 2001. A role of the gelatinous matrix in the resistance of root-knot nematode (Meloidogyne spp.) eggs to microorganisms. J. Nematol. 33:203-207.

19. Ornat, C., Verdejo-Lucas, S., and Sorribas, F. J. 2001. A population of Meloidogyne javanica in Spain virulent to the $M i$ resistance gene in tomato. Plant Dis. 85:271-276.

20. Prot, J. C. 1984. A naturally occurring resistance breaking biotype of 
Meloidogyne arenaria on tomato. Reproduction and pathogenicity on tomato cultivars Roma and Rossol. Rev. Nematol. 7:23-28.

21. Pinel, N., Davidson, S. K., and Sthal, D. A. 2008. Verminephrobacter eiseniae gen. nov., sp. nov., a nephridial symbiont of the earthworm Eisenia foetida (Savigny). Int. J. Syst. Evol. Microbiol. 58:2147-2157.

22. Roberts, P. A. 1995. Conceptual and practical aspects of variability in root-knot nematodes related to host-plant resistance. Annu. Rev. Phytopathol. 33:199-221.

23. Sacco, A. M., Koropacka, K., Grenier, E., Jaubert, M. J., Blanchard, A., Goverse, A., Smant, G., and Moffett, P. 2009. The cyst nematode SPRYSEC protein RBP-1 elicits GPa2-and RanGAP2-dependent plant cell death. PLoS Pathog. 5:1-14.

24. Sakai, H. 2010. A DNA extraction method with SDS from single nematodes for direct application to PCR amplification. Nematol. Res. 40:13-14

25. Schleheck, D., Knepper, T. P., Fischer, K., and Cook, A. M. 2004. Mineralization of individual congeners of linear alkylbenzenesulfonate by defined pairs of heterotrophic bacteria. Appl. Environ. Microbiol. 70:4053-4063.

26. Semblat, J. P., Bongiovanni, M., Wajnberg, E., Dalmasso, A., Abad, P., and Castagnone-Sereno, P. 2000. Virulence and molecular diversity of parthenogenetic root-knot nematodes, Meloidogyne spp. Heredity 84:81-89.
27. Semblat, J. P., Rosso, M. N., Hussey, R. S., Abad, P., and CastagnoneSereno, P. 2001. Molecular cloning of a cDNA encoding an amphidsecreted putative avirulence protein from the root-knot nematode Meloidogyne incognita. Mol. Plant-Microbe Interact. 14:72-79.

28. Verdejo-Lucas, S., Cortada, L., Sorribas, F. J., and Ornat, C. 2009. Selection of virulent populations of Meloidogyne javanica by repeated cultivation of Mi resistance gene tomato rootstocks under field conditions. Plant Pathol. 58:990-998.

29. Verdejo-Lucas, S., Cortada, L., Ornat, C., and Sorribas, F. J. 2010. Tomato rootstocks for management of Meloidogyne. 3rd International Symposium on Tomato Diseases. Ischia, Naples-Italy.

30. Williamson, V. M., Ho, J. Y., Wu, F. F., Miller, N., and Kaloshian, I. 1994. A PCR-based marker tightly linked to the nematode resistance gene $M i$ in tomato. Theor. Appl. Genet. 87:757-763.

31. Williamson, V. M. 1998. Root-knot nematode resistance genes in tomato and the potential for future use. Annu. Rev. Phytopathol. 36:277-293.

32. Williamson, V. M., and Gleason, C. A. 2003. Plant-nematode interactions. Curr. Opin. Plant Biol. 6:327-333.

33. Xu, J., Narabu, T., Mizukubo, T., and Hibi, T. 2001. A molecular marker correlated with selected virulence against the tomato resistance gene $M i$ in Meloidogyne incognita, M. javanica, and M. arenaria. Phytopathology 91:377-382. 